\title{
Payer Perspectives on Patient-Reported Outcomes in Health Care Decision Making: Oncology Examples
}

\author{
Andrew P. Brogan, PhD; Carla DeMuro, MS; Amy M. Barrett, MA, MSPH; Denise D'Alessio, MBA; \\ Vasudha Bal, MSc, MBA; and Susan L. Hogue, PharmD, MPH
}

\begin{abstract}
BACKGROUND: Health authorities and payers increasingly recognize the importance of patient perspectives and patient-reported outcomes (PROs) in health care decision making. However, given the broad variety of PRO endpoints included in clinical programs and variations in the timing of PRO data collection and country-specific needs, the role of PRO data in reimbursement decisions requires characterization.
\end{abstract}

OBJECTIVES: To (a) determine the effect of PRO data on market access and reimbursement decisions for oncology products in multiple markets and (b) assess the effect of PRO data collected after clinical progression on payer decision making.

METHODS: A 3-part assessment (targeted literature review, qualitative one-on-one interviews, and online survey) was undertaken. Published literature was identified through searches in PubMed/MEDLINE and Embase. In addition, a targeted search was conducted of health technology assessment (HTA) agency websites in the United States, the United Kingdom, France, and Germany. Qualitative one-on-one interviews were conducted with 16 payers from the RTI Health Solutions global advisory panel in 14 markets (Australia, Brazil, France, Germany, Italy, South Korea, Netherlands, Poland, Spain, Sweden, Taiwan, Turkey, the United Kingdom, and the United States $[n=3])$. Of the 200 payers and payer advisors from the global advisory panel invited to participate in the online survey, 20 respondents (China, France, Germany, Spain [ $n=2]$, Taiwan, the United Kingdom, and the United States $[n=13])$ completed the survey, and 6 respondents (Australia, South Korea, and the United States $[n=4]$ ) partially completed the survey.

RESULTS: Reviews of the literature and publicly available HTAs and reimbursement decisions suggested that HTA bodies and payers have varying experience with and confidence in PRO data. Payers participating in the survey indicated that PRO data may be especially influential in oncology compared with other therapeutic areas. Payers surveyed offered little differentiation by cancer type in the importance of PRO data but felt that it was most important to collect PRO data in phase 3 and postmarketing studies. Payers surveyed also anticipated an increasing significance for PRO data over the next 5-10 years. Characteristics of PRO data that maximize influence on payer decision making were reported to be (a) quality, well-controlled, and transparent PR0 evidence; (b) psychometric validation of the PR0 measure in targeted populations; and (c) publication in peer-reviewed journals. In markets with decentralized health care decision making, PRO data currently have more influence at the local level. Inclusion of PRO data in cancer treatment guidelines is key for centralized markets. Payers surveyed generally considered collecting PRO data postprogression to be useful. Of the 16 interviewees, 11 indicated that it is worthwhile to collect PRO data postprogression and that positive PRO data may support continued therapy at the physician's discretion upon regulatory approval, even in progressive disease. CONCLUSIONS: PRO data may help to differentiate treatments, particularly after clinical progression in oncology. Payers worldwide recognize highquality PRO data as a key component of their decision-making process and anticipate the growing importance of PRO data in the future.

J Manag Care Spec Pharm. 2017;23(2):125-34

Copyright $\odot 2017$, Academy of Managed Care Pharmacy. All rights reserved.

\section{What is already known about this subject}

Use of patient-reported outcome (PRO) measures in oncology clinical trials continues to increase.

There is lack of familiarity with PRO measures by some payers and a perceived lack of value of PRO data to support decision making. Use of PRO data by health technology assessment agencies varies, with oncology being a disease area that particularly utilizes PRO data.

\section{What this study adds}

Oncology is a therapeutic area in which PRO data can have a great effect given the high symptom burden and need for palliative care. To be fully considered by payers globally, PRO data should be collected using validated methods and published in peer-reviewed journals. For global payer consideration, postprogression PRO data are particularly important for cancer conditions that involve palliative and/or long-term care.

$\mathrm{P}$ atient-reported outcomes (PROs) —or outcomes reported directly by patients, without interpretation by clinicians or others ${ }^{1}$ - are an accepted and often actively solicited source of evidence used by health authorities and payers in evaluating and approving pharmaceutical interventions. ${ }^{2}$ PRO data are an important complement to the clinical evidence in demonstrating the value of a treatment, particularly for interventions developed to treat chronic, disabling conditions where the intention is not necessarily to cure but rather to alleviate symptoms, facilitate function, or improve quality of life..$^{2-4}$ However, owing to the numerous PRO endpoints that may be used in clinical trials, as well as variations in how payers in different markets assess evidence, the role of PRO data in reimbursement decisions is not well characterized.

The evaluation of new drugs for reimbursement and/or health technology assessment (HTA) involves developing recommendations, typically of greatest interest to payers, that incorporate clinical information about a treatment's effectiveness and economic information about a treatment's value. In addition, there is growing recognition that the patient perspective is an important component of HTAs. ${ }^{2}$ Patient input is an important factor when assessing the economic, social, and ethical implications of the approval and use of a treatment. As such, it is expected that PROs will be included increasingly in 
HTAs and will influence market access, reimbursement, and pricing negotiations. ${ }^{2}$

Although the importance of PROs in characterizing the treatment impact is generally well recognized, the presence of PRO data in oncology product labeling and the collection of data after clinical progression is somewhat uncommon. Survival and other clinical outcomes tend to be emphasized as primary and key secondary endpoints over PROs in oncology drug development programs, despite the significant toxicities that may be conferred by cancer drugs and their substantial effect on patients' health-related quality of life. ${ }^{5}$ However, PRO data in oncology studies provide valuable information on the patient experience beyond product labeling, which is increasingly a factor in the HTA process. Thus, we sought to characterize payers' impressions of oncology-related PRO data by conducting a literature review, one-on-one interviews, and an online survey with a sample of global payers, a method previously employed in qualitative research with payers. ${ }^{6}$ The objectives for this study were to (a) determine the effect of PRO data on market access and reimbursement decisions for oncology products in multiple markets and (b) assess the effect of PRO data collected after clinical progression (i.e., postprogression) on payer decision making.

\section{Methods}

To obtain insights into the experience of HTA bodies and payers with PRO data and to help inform the development of a semistructured interview guide for the one-on-one interviews with payers and an online survey instrument, we conducted a targeted review of the literature. Searches of the PubMed/ MEDLINE and Embase databases were undertaken in May 2014 using broad search terms relating to PROs, oncology, and technology assessment or reimbursement. No date limits were included in the searches. In addition, we searched the websites of 9 agencies for HTAs in which PRO data appeared to have been included. These agencies were the Pharmaceutical Benefits Advisory Committee (Australia), Canadian Agency for Drugs and Technologies in Health, Haute Autorité de Santé (HAS), Institute for Quality and Efficiency in Health Care (IQWiG), Scottish Medicines Consortium, National Health Service Scotland (NHS Scotland), National Institute for Health and Care Excellence, Drug Effectiveness Review Project (United States), and Agency for Healthcare Research and Quality (United States). Drugs manufactured by the study sponsor were excluded from both searches.

Payers were recruited from the RTI Health Solutions global advisory panel for one-on-one interviews (August 1, 2014October 31, 2014) and an online survey (December 8, 2014March 4, 2015). The information collected during the interviews included the roles of the participants in the oncology treatment chain, as well as their impressions of the role of PRO measures in market access decisions, the characteristics or qualities that PRO measures must have to influence HTA and market access decisions, and other considerations for the use of PRO measures in oncology. In addition, a web-based survey was developed to collect information similar to what was captured in the one-on-one interviews; however, the online survey was administered to a broader international sample of payers and payer advisors. The full survey instrument is presented in Appendix A (available in online article). Survey invitations were sent to 200 payers and payer advisors from the RTI Health Solutions global payer advisory panel. Survey responses were collected from December 8, 2014, to March 4, 2015.

\section{Results}

\section{Literature Review}

A total of 21 sources were identified for full-text review from the searches of the literature databases and HTA sites. Of these, 9 sources provided summary information on the use of PROs in oncology HTAs. Two specific references provided key information that was particularly relevant for the primary payer research: a multinational review of HTAs and a review of the impact of oncology-related PROs on payer decision making in the United States. ${ }^{7,8}$ In addition, the following 4 drugs had at least 1 HTA that included PRO data: bevacizumab (Avastin, Roche/Genentech), pemetrexed (Alimta, Eli Lilly and Company), sunitinib (Sutent, Pfizer), and crizotinib (Xalkori, Pfizer; Table 1).

To determine the prevalence of PRO data in HTAs, Rubinstein et al. (2012) reviewed 324 HTAs issued by 9 agencies between 2005 and 2011 in 13 disease areas, including 2 types of cancer (ovarian and chronic myelogenous leukemia) and anemia in cancer. ${ }^{7}$ Of the 324 HTAs reviewed, 91 (28\%) reported PRO data. The use of PRO data increased over time, from $11.1 \%$ in 2005 to $42.5 \%$ in 2011. For several disease conditions, PROs were not used; however, for ovarian cancer and anemia in cancer, the HTAs reviewed used PROs at least $75 \%$ of the time. Use of PRO data varied by HTA body, from $9.8 \%$ in HAS HTAs to $66.7 \%$ in HTAs by IQWiG and NHS Scotland. ${ }^{7}$

Similarly, Zagadailov et al. (2013) found that the use of PRO measures in oncology trials, along with literature describing the development, interpretation, and incorporation of PRO measures into oncology studies, has increased sharply in recent years. ${ }^{8}$ They credited much of the increased interest in and frequency of oncology PRO measures to the release of the U.S. Food and Drug Administration's (FDA) PRO guidance document. ${ }^{1}$ Nevertheless, Zagadailov et al. found that U.S. payer decision makers have varying levels of familiarity and confidence with PRO data. These factors support the need for payers to continue to become familiarized with PRO measures to fully appreciate the risk-benefit profile of novel agents. ${ }^{8}$ 
Payer Perspectives on Patient-Reported Outcomes in Health Care Decision Making: Oncology Examples

TABLE 1 Oncology-Related PROs in HTAs

\begin{tabular}{|c|c|c|}
\hline Country: HTA Agency (Reference) & Decision & Key Comments \\
\hline \multicolumn{3}{|l|}{ Bevacizumab } \\
\hline United Kingdom: NICE ${ }^{13-16}$ & $\begin{array}{l}\text { Reviewed and rejected in multiple } \\
\text { oncology indications }\end{array}$ & $\begin{array}{l}\text { "Health-related quality-of-life data collected in the trial would have } \\
\text { been preferable for deriving the utilities for the economic model." } 13\end{array}$ \\
\hline France: HAS17 & Unrestricted reimbursement & $\begin{array}{l}\text { "Patient quality of life was not analyzed for the totality of question- } \\
\text { naires (various data were found to be missing) and, as this was an } \\
\text { open-label study, the results of this analysis can therefore not be } \\
\text { taken into consideration in this opinion."17 }\end{array}$ \\
\hline \multicolumn{3}{|r|}{ 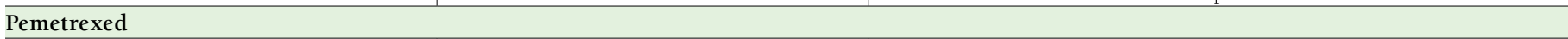 } \\
\hline United Kingdom: NICE ${ }^{18}$ & $\begin{array}{l}\text { Recommended for malignant pleural } \\
\text { mesothelioma and NSCLC }\end{array}$ & $\begin{array}{l}\text { Committee noted that, over } 18 \text { weeks, patients with NSCLC treated } \\
\text { with pemetrexed plus cisplatin demonstrated statistically signifi- } \\
\text { cant symptomatic improvements when compared with those who } \\
\text { received cisplatin alone in Lung Cancer Symptom Scale-Meso. }{ }^{18}\end{array}$ \\
\hline \multicolumn{3}{|l|}{ Sunitinib } \\
\hline United Kingdom: NICE ${ }^{19}$ & Recommended for RCC & $\begin{array}{l}\text { "There is a paucity of data on the utility values associated with living } \\
\text { with advanced and/or metastatic RCC...it may be difficult to fully } \\
\text { capture the effects of sunitinib on health-related quality of life."19 }\end{array}$ \\
\hline \multicolumn{3}{|l|}{ Crizotinib } \\
\hline Germany: IQWiG20 & $\begin{array}{l}\text { 2012: showed no added benefit for } \\
\text { patient-relevant endpoints for ALK- } \\
\text { positive NSCLC } \\
\text { 2013: showed added benefit after spon- } \\
\text { sor submitted additional data related to } \\
\text { symptoms (morbidity) and HRQOL }\end{array}$ & $\begin{array}{l}\text { Added benefit of crizotinib for HRQOL was rated "considerable" } \\
\text { based on results from the EORTC QLQ-C30, EORTC QLQ-LC13, } \\
\text { and EQ-5D. }{ }^{20}\end{array}$ \\
\hline
\end{tabular}

ALK = anaplastic lymphoma kinase; EORTC QLQ = European Organization for Research and Treatment of Cancer Quality of Life Questionnaire; EQ-5D = measure of health outcome; HAS = Haute Autorité de Santé; HRQOL = health-related quality of life; HTA=health technology assessment; IQWiG =Institute for Quality and Efficiency in Health Care; NICE= National Institute for Health and Care Excellence; NSCLC =non-small cell lung cancer; PRO=patient-reported outcome; RCC = renal cell carcinoma.

\section{Primary Research with Global Payers}

Sample Characteristics. Qualitative one-on-one interviews were conducted with 16 payers and payer advisors in 14 markets (Australia, Brazil, France, Germany, Italy, South Korea, Netherlands, Poland, Spain, Sweden, Taiwan, Turkey, the United Kingdom, and the United States [n=3]). Of the 200 payers and payer advisors invited to participate in the survey, 20 respondents (from China, France, Germany, Spain $[\mathrm{n}=2]$, Taiwan, the United Kingdom, and the United States $[\mathrm{n}=13])$ completed the survey, and an additional 6 respondents (from Australia, South Korea, and the United States $[n=4]$ ) partially completed the survey. Table 2 presents the sample characteristics. The qualitative interview participants and the survey respondents were primarily medical and pharmacy directors and HTA advisors. The interviews and survey were all conducted in English.

When asked about their roles in the treatment chain for oncology, the interview participants and survey respondents described themselves as making decisions regarding formulary recommendations ( $46 \%$ and $31 \%$, respectively) or advising decision makers on whether a treatment is a good value $65 \%$ and $81 \%$, respectively) or appropriate for use based on clinical factors (31\% and 38\%, respectively).

Role of PROs Data in Market Access. Payer participants interviewed generally indicated that they consider PRO evidence in their decisions but that such evidence is usually considered complementary to clinical and safety endpoints. PRO evidence appears to have greater influence outside the United States: When asked whether PRO data affect decision making when one therapy is being considered over another, $47 \%$ of U.S. payers and $78 \%$ of ex-U.S. payers in the web-based survey indicated that PRO data have an impact. In addition, payers participating in the one-on-one interviews generally indicated that PRO data had greater weight in major markets (e.g., Australia, France, Germany, the Netherlands, Sweden, the United Kingdom, and the United States) than in developing markets (e.g., Brazil, China, South Korea, Poland, Taiwan, and Turkey) and markets under particular budgetary constraints owing to the recent financial crisis (e.g., Italy and Spain). The interviewed payers also generally noted that the role of PRO evidence in market access and uptake differs by market type. In decentralized markets (e.g., Brazil, Italy, Spain, Taiwan, and the United States), PRO evidence has the greatest effect at the local level, influencing physicians' prescribing habits and, in turn, potentially affecting patient uptake of a therapy. In centralized markets (e.g., France, Germany, Netherlands, and the United Kingdom), PRO data have an effect on national-level assessment and only a minimal effect at regional and local levels. Payers interviewed also noted that incorporation of PRO data in national and international cancer treatment guidelines is a key factor in the effect of PRO data on decision making in centralized markets. 


\section{TABLE 2 Sample Characteristics}

\begin{tabular}{|c|c|c|c|c|c|}
\hline \multicolumn{6}{|c|}{ Qualitative One-on-One Interview Participants } \\
\hline Country & \multicolumn{5}{|c|}{ Payer Advisor Profile } \\
\hline Australia & \multicolumn{5}{|c|}{ Advisor to MSAC and PBAC } \\
\hline Brazil & \multicolumn{5}{|c|}{ Clinical oncologist, professor, and advisor to private insurance providers } \\
\hline France & \multicolumn{5}{|l|}{ Advisor to HAS } \\
\hline Germany & \multicolumn{5}{|c|}{ Member of the arbitration board for drug process in the statutory health insurance } \\
\hline Italy & \multicolumn{5}{|c|}{ Health economics professor and advisor to a regional health agency (Lombardo/Lazio local/hospital) } \\
\hline South Korea & \multicolumn{5}{|c|}{ Advisor to Health Insurance Review and Assessment (HIRA) } \\
\hline Netherlands & \multicolumn{5}{|c|}{ Advisor to Zorginstituut Nederland (ZINL, formerly CVZ) } \\
\hline Poland & \multicolumn{5}{|c|}{ Advisor to Agenca Oceny Technologii Medycznych (AOTM) } \\
\hline Spain & \multicolumn{5}{|c|}{ Health economics professor and advisor to regional health authorities (Andalucia) } \\
\hline Sweden & \multicolumn{5}{|c|}{ Advisor to Tandvårds-och läkemedelsförmånsverket (TVL) } \\
\hline Taiwan & \multicolumn{5}{|l|}{ HTA advisor } \\
\hline Turkey & \multicolumn{5}{|c|}{ Advisor to public and private insurance providers } \\
\hline United Kingdom & \multicolumn{5}{|c|}{ Advisor to NICE } \\
\hline \multicolumn{6}{|l|}{ United States } \\
\hline \multirow[b]{2}{*}{ Position } & \multirow{2}{*}{$\begin{array}{l}\text { Geographic } \\
\text { Coverage Area }\end{array}$} & \multicolumn{4}{|c|}{ Covered Lives } \\
\hline & & Total (Millions) ${ }^{a}$ & Commercial (\%) & Medicare (\%) & Medicaid (\%) \\
\hline Medical director & National & 11.0 & 17 & 43 & 5 \\
\hline Pharmacy director & National & 35.0 & 70 & 15 & 15 \\
\hline Medical director & Employer payer & 0.5 & 100 & 0 & 0 \\
\hline \multicolumn{6}{|c|}{ Online Survey Participants } \\
\hline Country & Surve & ipleted & \multicolumn{3}{|c|}{$\begin{array}{c}\text { Surveys Partially Completed } \\
\text { (Last Completed Question of } 15 \text { Questions) }\end{array}$} \\
\hline Australia & \multicolumn{2}{|c|}{0} & \multicolumn{3}{|c|}{$1(\mathrm{Q} 10)$} \\
\hline China & \multicolumn{2}{|c|}{1} & \multicolumn{3}{|c|}{0} \\
\hline France & \multicolumn{2}{|c|}{1} & \multicolumn{3}{|c|}{0} \\
\hline Germany & \multicolumn{2}{|c|}{1} & \multicolumn{3}{|c|}{0} \\
\hline South Korea & \multicolumn{2}{|c|}{0} & & $1(\mathrm{Q} 2)$ & \\
\hline Spain & & & & 0 & \\
\hline Taiwan & & & & 0 & \\
\hline United Kingdom & & & & 0 & \\
\hline United States & 5 pharmacy direct & edical directors & $\begin{array}{l}1 \text { pharmacy directo } \\
\text { respectively) }\end{array}$ & 3 medical dire & Q4, and Q12, \\
\hline
\end{tabular}

a35\% Tricare.

HAS = Haute Autorité de Santé; HTA = health technology assessment; MSAC= Medical Services Advisory Committee; NICE= National Institute for Health and Care Excellence; PBAC= Pharmaceutical Benefits Advisory Committee.

PRO evidence was seen as a moderately important factor in market access for new oncology treatments by payers who were surveyed (Figure 1A). However, payers surveyed inside and outside the United States indicated that the importance of PRO data for reimbursement decisions would increase in the next 5 years. Specifically, it is anticipated that PRO evidence could become a key differentiator among treatments as payers increasingly consider value in their decision making. A U.S. payer commented that robust PRO data for an oncology product may be more meaningful than modest survival gains. It was also noted that PRO evidence does not currently have the weight of clinical evidence in reimbursement decision making. but it was suggested that PRO data could emerge as an extremely important factor within the next decade.
Payer participants generally noted that PRO data currently are used infrequently in risk-sharing agreements, particularly in U.S. plans: $75 \%$ of U.S. payer participants and 38\% of ex-U.S. payers indicated that PRO data are never used in risk-sharing agreements, with no payers indicating that PRO data are always used in risk-sharing agreements. However, as risk-sharing agreements become more prevalent globally, some payer interviewees suggested that PRO data could factor into risk-sharing agreements in the future.

Characteristics of PROs with the Greatest Influence on Reimbursement. Payer interviewees generally indicated that there are minimal requirements or guidelines specifically addressing whether and how payer decision makers use PRO evidence. Instead, PRO evidence is typically evaluated on a 
A. Mean Importance of PRO Data in General, Currently and in 5 Years

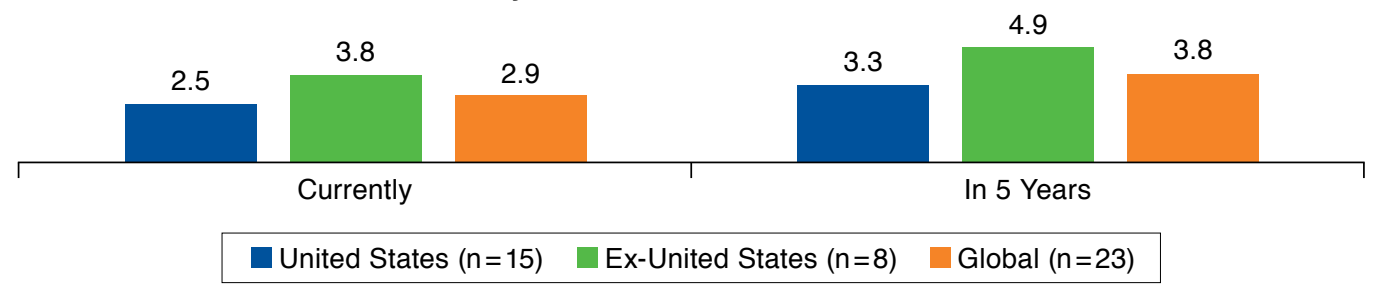

\section{B. Usefulness of Postprogression PRO Data}

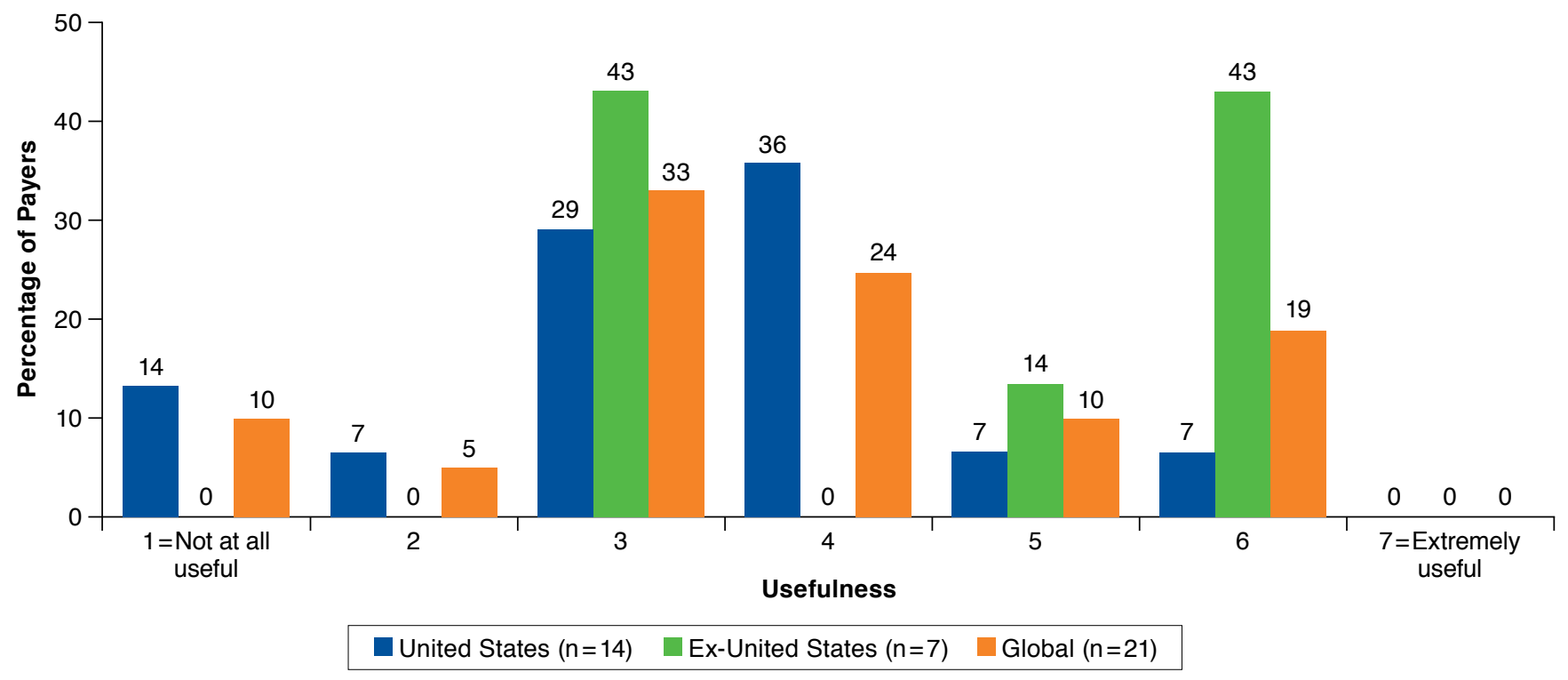

amportance/usefulness was reported using a scale of 1 (not important/useful at all) to 7 (extremely important/useful). $P R O=$ patient-reported outcome.

case-by-case basis (e.g., relevance and transparency), and the quality of the PRO evidence is paramount. Figure 2 presents the characteristics that payers recommended for PRO measures to have in order to support market access and HTAs for an oncology product. Payers in all markets reported in this study consistently noted the importance of appropriate validation for PRO measures and dissemination of PRO data in peer-reviewed publications.

When asked about the importance of the validation of PRO measures, most $(83 \%)$ payers suggested that the effect of PRO data can be marginalized if a measure is not fully validated in the target disease population-if, for example, the measure is newly developed. The majority (63\%) of ex-U.S. payers also emphasized the importance of validation in a country-specific population (Figure 2). A majority (73\%) of U.S. payers also indicated that PRO measures should be developed, evaluated, and validated in accordance with the FDA's PRO guidance document, ${ }^{1}$ although it should be noted that many payer participants were unfamiliar with the specific criteria presented in the guidance. Most (80\%) U.S. payers also indicated that they value PRO measures that align with objective measures, such as laboratory values and clinician ratings.

Although PRO evidence may be reported in product labeling and/or in peer-reviewed publications, payer participants generally indicated that PRO claims in the product label have more of an effect on reimbursement for a treatment outside the United States (Appendix B, available in online article); however, payer interviewees also suggested that U.S. payer decision making may be affected through increased awareness of and education about PRO label claims in the future. When asked whether PRO data in product labeling or in peerreviewed publications have a greater effect on decision making for a new treatment, a majority (60\%) of U.S. payer participants and a considerable majority (75\%) of ex-U.S. payer participants 
indicated that PRO data in peer-reviewed publications would be more influential (Figure 3A).

Payer participants were asked whether the evaluation process would be different depending on whether PRO data were from registration trials or from postmarketing studies. Across markets, at least $50 \%$ of survey participants indicated that PRO data from registration trials and postmarketing studies are thought to be equally important and useful (Figure 3B). In the United States, 31\% of payer participants indicated that PRO data from postmarketing studies are held in higher regard or are more useful than data from registration trials. A German payer noted that postmarketing PRO data are especially useful for reevaluation in Europe and for appealing negative decisions (e.g., crizotinib in Germany).

Considerations for PRO Measures in Oncology. All oneon-one interview participants indicated that it is worthwhile to collect PRO data in oncology clinical trials, and oncology was ranked as the therapeutic area in which PROs had the greatest influence on decision making (more so than in other areas, including neurology, psychiatry, metabolic disease, and lifestyle-related conditions such as obesity and smoking). When asked about any specific oncology products with PRO data that particularly affected favorable reimbursement decisions, U.S. payers cited enzalutamide for prostate cancer and ruxolitinib for myelofibrosis as products whose reimbursement status benefited from the inclusion of PRO data. In addition, a German payer noted that PRO data were key in crizotinib having received an additional benefit rating under AMNOG (Act on the Reform of the Market for Medical Products [Arzneimittelmarkt-Neuordnungsgesetz]), a crucial step for a product to achieve any price premium in Germany.

Payer participants across markets considered PRO data for an oncology treatment collected after clinical progression to be moderately useful, and in general, postprogression PRO 
A. Percentage of Payers Indicating Greater Effect of PRO Data in Product Labeling Versus in Peer-Reviewed Publication

United States $(n=15)$

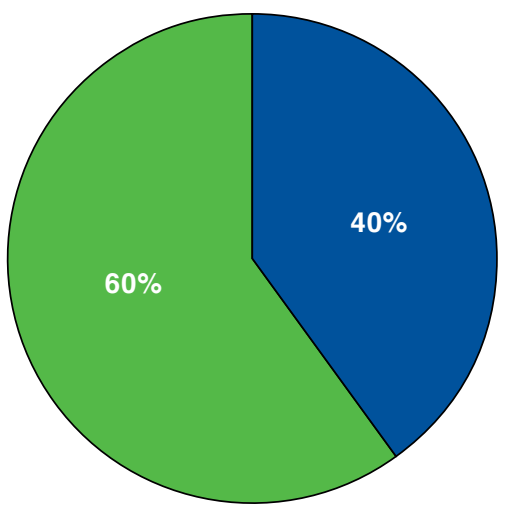

Ex-United States $(n=8)$

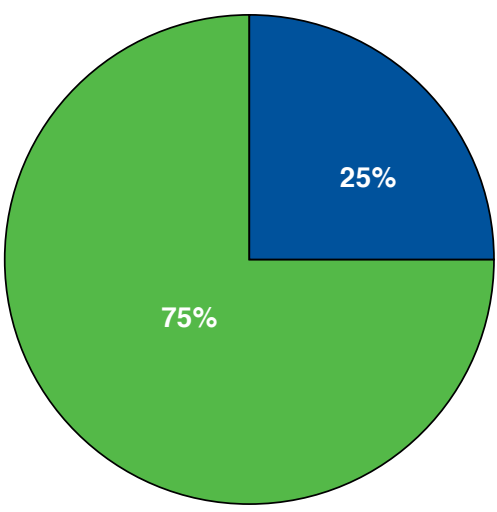

Global $(n=23)$

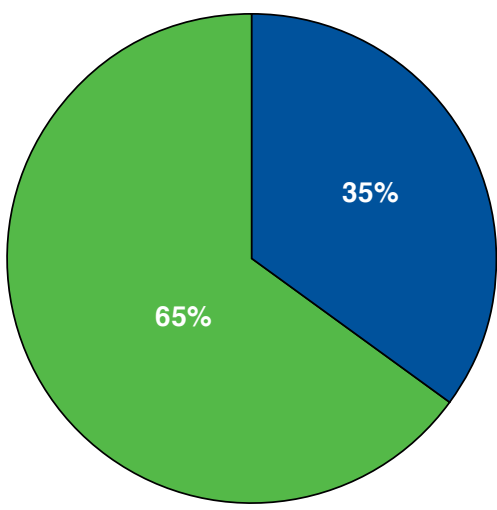

PRO data in the label PRO data in a peer-reviewed publication

B. Percentage of Payers Indicating Greater Effect of PRO Data from Registration Trials Versus Postmarketing Studies
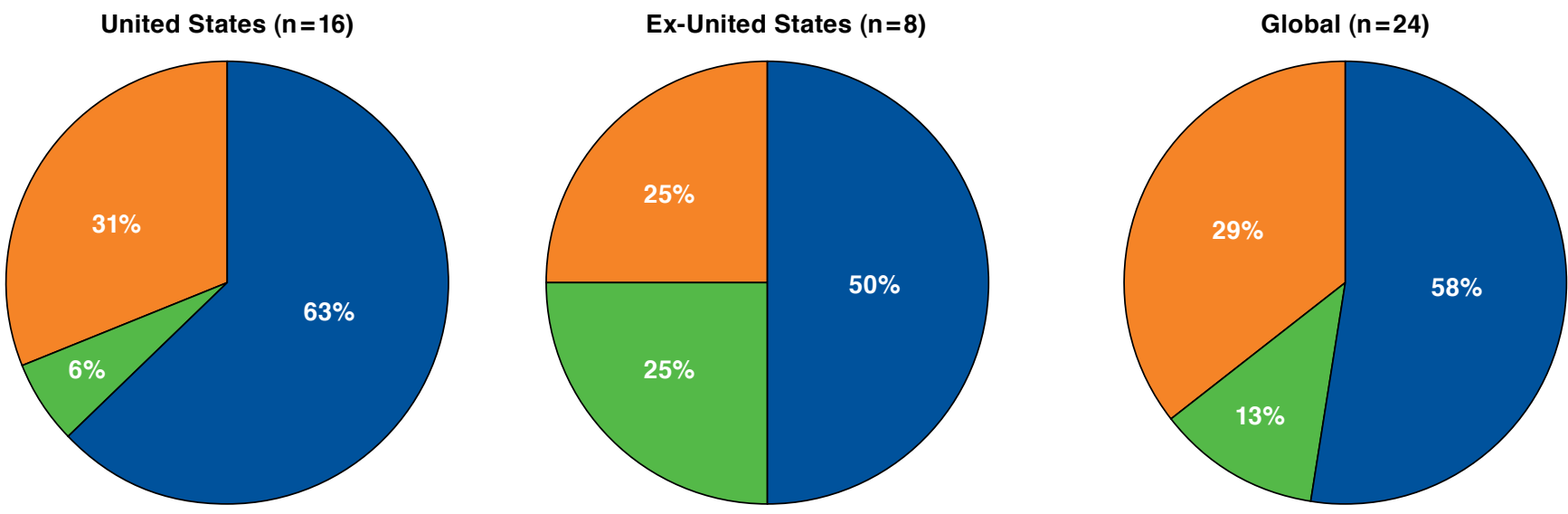

Same for both types of trials Higher regard/more useful information from registration trials

Higher regard/more useful information from postmarketing trials

$P R O=$ patient-reported outcome.

data were considered more useful in ex-U.S. markets than in U.S. markets (Figure 1B). Although payers did not have strong preferences for specific cancer indications in which postprogression PRO data should be collected, the majority (68\%) of participants in the one-on-one interviews indicated that postprogression data are particularly important for cancer conditions that involve palliative and/or long-term care (e.g., lung cancer, breast cancer, and bladder cancer). In addition, the majority (68\%) of one-on-one interviewees indicated that positive postprogression PRO data may support further use of the therapy at the physician's discretion upon regulatory approval, even in progressive disease. It was also suggested that PRO data may help differentiate treatments postprogression and could affect decision making, particularly in the future.

Payers were asked to rate which outcomes (stability of disease, improvement in health-related quality of life, improvement in symptom severity or frequency, improvement in functional status, slowing of functional deterioration relative to comparator, or other) would be most convincing for supporting appropriate reimbursement of oncology treatments 
postprogression. Ex-U.S. and U.S. payers consistently rated improvements in health-related quality of life and functional status as being extremely important, and U.S. payers also rated improvements in symptom severity or frequency as extremely important.

Several payer interviewees commented on the need for the industry to educate clinicians and payers about PRO data. Some HTA agencies (e.g., Health Insurance Review and Assessment Service in South Korea) convene clinician panels to advise on the assessment process, and the efforts of manufacturers to clarify PRO evidence for clinicians may improve payers' understanding of PRO data. One payer participant suggested that prescribers focus primarily on clinical outcomes in oncology, such as tumor progression and progression-free survival and may not be familiar with PRO measures and data. Accordingly, another participant recommended that manufacturers integrate PRO data with clinical outcomes as part of a broader value proposition. It was suggested that PRO data should demonstrate patient-relevant benefits, should be based on existing validated measures, and should be evaluated relative to an appropriate comparator (as determined by an HTA agency).

\section{Discussion}

This study provides a contemporary, global perspective on the value of PRO data in HTAs with a focus on oncology. Payers participating in one-on-one interviews and an online survey indicated that the inclusion of PRO data in reimbursement decision making varies by country and within country by payer type (i.e., national, regional, or local decision makers). Our findings suggest that there is a growing recognition of the value of quality PRO data in the oncology area and that postprogression PRO data in particular may have an immediate impact on HTA decision making. PRO evidence also may have a role in risk-sharing agreements as such agreements become more prevalent globally. Effective PRO data should be collected in either registration trials or postmarketing studies using measures that have been validated for the appropriate target disease population. For providers, payers, and other stakeholders to understand the value of PRO data, publication in peerreviewed journals is critical. In addition, PRO data relating to a new treatment should be considered as part of a larger value proposition.

The concept of value with respect to cancer treatments is evolving: the American Society of Clinical Oncology value framework, for example, acknowledges the relevance of PRO data in considerations of net health benefit for oncology drugs and suggests that future value frameworks should incorporate reliable, consistently collected, and evidence-based PRO data. ${ }^{9}$ Patient-centered data are also being incorporated into clinical practice, and treatment guidelines may recommend the assessment of pain and other PROs. ${ }^{10}$ Stakeholders increasingly are considering how outcomes such as symptom benefits or patient functioning may be influenced as a result of treatment. Our findings provide context for how PRO data may influence market access and health care decision making for new oncology products, and payer insights with regard to postprogression data in particular may have implications for future clinical study designs.

Two previous studies surveying U.S. payers, HTA advisors, and policymakers noted poor familiarity with PRO measures and low levels of confidence in the quantity and quality of the data and its value in decision making. ${ }^{8,11}$ Participants in one of these studies noted that, although PRO data may be useful in differentiating treatments with similar efficacy or comparable toxicity profiles, state mandatory coverage regulations or regulations from the Centers for Medicare \& Medicaid Services may prohibit a payer from designative coverage preference for one drug over another regardless of seemingly superior PRO data. ${ }^{8}$ However, despite these challenges, it was noted that PRO data will increasingly affect drug selection decisions made by individual patients and their health care providers. ${ }^{8}$

A review of HTAs has shown wide variation in the use of PRO data, from as low as 10\% (HAS) to approximately $68 \%$ (IQWiG and NHS Scotland). ${ }^{7}$ However, between 2005 and 2011, assessments in oncology disease conditions increasingly incorporated PRO evidence, suggesting that PRO data are emerging as an important factor in HTAs of cancer therapies. ${ }^{7}$ A review of breast cancer and colorectal cancer medicines in a selection of industrialized markets (Canada, Australia, the United Kingdom, France, and Scotland) identified 3 primary differences in approach to HTA that appear to drive recommendations: (a) differences in the methods used to interpret surrogate endpoints (e.g., progression-free survival and disease-free survival were judged to be acceptable vs. overall survival); (b) differences in the extent to which agencies considered patient voice or perspective; and (c) differences in what was considered an appropriate comparator. ${ }^{12}$

Manufacturers should educate payers and prescribers on the PRO data pertinent to their therapies and tie the data to the broader value story for new therapies introduced to the market. Insights into the patient experience may have an effect on payer and prescriber decision making. PRO data can provide value for differentiation versus competitors, in turn influencing prescribing patterns and local- and national-level decision making. PROs can be integral to broader market access if tied to a patient-centric value proposition, but a strong a priori communication strategy is critical. Differences in the extent to which agencies consider patient voice or perspective may lead to different decisions for the same product. 


\section{Limitations}

This study was limited by the number of markets represented and the number of participants per market, particularly outside the United States. Nevertheless, payer participants provided a global perspective. Other well-designed qualitative studies have also identified important payer perspectives based on interviews with a similarly sized sample (e.g., Handfield and Feldstein $\left.[2013]^{6}\right)$. The low response rate for the survey also should be considered when the results are interpreted: representativeness of the sample could not be ensured, and the findings may not be generalizable to the entire payer population. Finally, the survey instrument and interview discussion guide used to elicit participant input were not validated questionnaires.

\section{Conclusions}

The importance of PRO data in HTAs and market access for oncology therapies is increasing. To support payer decision making, well-developed and validated PRO measures should be incorporated into studies of oncology therapies, and PROs should be tied to "actionable" steps that can be taken by clinicians and/or payers. Efforts should be made to publish PRO data and to educate prescribers and payers regarding PRO data and their value in health care decision making.

\section{Authors}

ANDREW P. BROGAN, PhD; CARLA DEMURO, MS; AMY M. BARRETT, MA, MSPH; and SUSAN L. HOGUE, PharmD, MPH, RTI Health Solutions, Research Triangle Park, North Carolina. DENISE D'ALESSIO, MBA, and VASUDHA BAL, MSc, MBA, Novartis Pharmaceuticals, East Hanover, New Jersey.

AUTHOR CORRESPONDENCE: Susan L. Hogue, PharmD, MPH, RTI Health Solutions, 200 Park Offices Dr., Research Triangle Park, NC 27709. Tel.: 919.541.6213; E-mail: shogue@rti.org.

\section{DISCLOSURES}

This study and preparation of this article were funded by Novartis Pharmaceuticals. This research was performed under a research contract between RTI Health Solutions and Novartis Pharmaceuticals. Brogan, Hogue, Demuro, and Barrett are employees of RTI Health Solutions. D'Alessio and Bal are employees of Novartis Pharmaceuticals.

Study concept and design were contributed by DeMuro, Barrett, Bal, and Hogue. Brogan and Hogue took the lead in data collection, assisted by DeMuro and Bal. Data interpretation was performed by Brogan and Hogue, assisted by the other authors. The manuscript was written by D'Alessio and Brogan, along with the other authors, and revised primarily by Brogan, along with Hogue and assisted by the other authors.
The abstract for this article was presented as a research poster at the following meetings:

- Hogue SL, Brogan AP, De Muro C, D’Alessio D, Bal V. Patient-reported outcomes (PRO) in post-progression oncology: implications in health technology assessments and payer decision making. Poster presented at the ISPOR 18th Annual European Meeting; November 7-11, 2015. Milan, Italy.

- Hogue SL, Brogan AP, De Muro C, D’Alessio D, Bal V. Influence of patientreported outcomes (PRO) on market access decisions in markets with centralized healthcare systems. Poster presented at the ISPOR 18th Annual European Meeting; November 7-11, 2015. Milan, Italy.

- Hogue SL, Brogan AP, De Muro C, Barrett A, D’Alessio D, Bal V. Influence of patient-reported outcomes on market access decisions in decentralized markets (Brazil, Italy, Spain and the United States). Poster presented at the ISPOR 20th Annual Meeting; May 16-20, 2015. Philadelphia PA.

- Hogue SL, Brogan AP, De Muro C, Barrett A, McLeod L, D'Alessio D, et al. Payer Perspectives of Patient-Reported Outcomes Data: An Online Assessment. Poster presented at the ISOQOL 22nd Annual Meeting; October 21-24, 2015. Vancouver, British Columbia, Canada.

\section{ACKNOWLEDGMENTS}

Kate Lothman of RTI Health Solutions provided medical writing services, which were funded by Novartis Pharmaceuticals.

\section{REFERENCES}

1. U.S. Food and Drug Administration. Guidance for industry. Patientreported outcome measures: use in medical product development to support labeling claims. December 2009. Available at: http://www.fda.gov/downloads/Drugs/.../Guidances/UCM193282.pdf. Accessed December 19, 2016.

2. Doward LC, Gnanasakthy A, Baker MG. Patient reported outcomes: looking beyond the label claim. Health Qual Life Outcomes. 2010;8:89.

3. DeMuro C, Clark M, Mordin M, Fehnel S, Copley-Merriman C, Gnanasakthy A. Reasons for rejection of patient-reported outcome label claims: a compilation based on a review of patient-reported outcome use among new molecular entities and biologic license applications, 2006-2010. Value Health. 2012;15(3):443-38

4. Gnanasakthy A, Mordin M, Clark M, DeMuro C, Fehnel S, CopleyMerriman C. A review of patient-reported outcome labels in the United States: 2006 to 2010. Value Health. 2012;15(3):437-42.

5. Basch E, Geoghegan C, Coons SJ, et al. Patient-reported outcomes in cancer drug development and US regulatory review: perspectives from industry, the Food and Drug Administration, and the patient. JAMA Oncol. 2015;1(3):375-79.

6. Handfield R, Feldstein J. Insurance companies' perspectives on the orphan drug pipeline. Am Health Drug Benefits. 2013;6(9):589-98.

7. Rubinstein E, Jaksa A, Ho YS, Daniel K. Use of PRO analysis in health technology assessments [Abstract PIH55]. Value Health. 2012;15(4):A201.

8. Zagadailov E, Fine M, Shields A. Patient-reported outcomes are changing the landscape in oncology care: challenges and opportunities for payers. Am Health Drug Benefits. 2013;6(5):264-74.

9. Schnipper LE, Davidson NE, Wollins DS, et al. Updating the American Society of Clinical Oncology Value Framework: revisions and reflections in response to comments received. J Clin Oncol. 2016;34(24):2925-34.

10. American Urological Association. Castration-resistant prostate cancer: AUA guideline for urologists. 2013. Available at: https://www.auanet.org/ common/pdf/education/clinical-guidance/Castration-Resistant-ProstateCancer-PG.pdf. Accessed December 19, 2016.

11. Svoboda K, White N. Payer evidence requirements for new drugs: trends and impact. Am J Manag Care. Published online April 4, 2014. Available at: http://www.ajmc.com/conferences/AMCPSpring2014/Payer-EvidenceRequirements-for-New-Drugs-Trends-and-Impact. Accessed December 19, 2016 
12. Shah KK, Mestre-Ferrandiz J, Towse A, Smyth EN. A review of health technology appraisals: case studies in oncology. Int J Technol Assess Health Care. 2013;29(1):101-09.

13. National Institute for Health and Care Excellence (NICE). Bevacizumab in combination with paclitaxel and carboplatin for first-line treatment of advanced ovarian cancer. Technology appraisal guidance [TA284]. May 22, 2013. Available at: http://www.nice.org.uk/guidance/TA284/chapter/lguidance. Accessed December 19, 2016.

14. National Institute for Health and Care Excellence (NICE). Bevacizumab in combination with capecitabine for the first-line treatment of metastatic breast cancer. Technology appraisal guidance [TA263]. August 22, 2012. Available at: https://www.nice.org.uk/Guidance/ta263. Accessed December 19, 2016.

15. National Institute for Health and Care Excellence (NICE). Cetuximab, bevacizumab and panitumumab for the treatment of metastatic colorectal cancer after first-line chemotherapy. Technology appraisal guidance [TA242]. January 25, 2012. Available at: https://www.nice.org.uk/Guidance/TA242. Accessed December 19, 2016.

16. National Institute for Health and Care Excellence (NICE). Bevacizumab in combination with a taxane for the first-line treatment of metastatic breast cancer. Technology appraisal guidance [TA214]. February 23, 2011. Available at: https://www.nice.org.uk/Guidance/TA214. Accessed

December 19, 2016.
17. Haute Autorité de Santé (HAS). AVASTIN CT 5075: Extension of indication of first-line therapy in patients with metastatic breast cancer, in combination with paclitaxel. December 5, 2007. Available at: http://www.hassante.fr/portail/upload/docs/application/pdf/2010-01/avastin_en_ct__5075_ def.pdf. Accessed December 19, 2016.

18. National Institute for Health and Care Excellence (NICE). Pemetrexed for the treatment of malignant pleural mesothelioma. Technology appraisal guidance [TA135]. January 23, 2008. Available at: https://www.nice.org.uk/ guidance/TA135. Accessed December 19, 2016.

19. National Institute for Health and Care Excellence (NICE). Sunitinib for the first-line treatment of advanced and/or metastatic renal cell carcinoma. Technology appraisal guidance [TA169]. March 25, 2009. Available at: https://www.nice.org.uk/guidance/TA169. Accessed December 19, 2016.

20. Institute for Quality and Efficiency in Health Care (IQWiG). Addendum to Commission A12-15 (crizotinib). IQWiG Reports - No. 162. April 15, 2013.

Available at: https://www.iqwig.de/download/A13-13_Addendum-toCommission-A12-15_crizotinib.pdf. Accessed December 19, 2016. 


\section{APPENDIX A Survey Questions}

Question

1. What is your role in the treatment chain for oncology? There are several roles that may apply. Please check all that apply.

Response Option

- Make prescribing decisions for individual patients (e.g., treating physician)

- Make decisions regarding which of 2 or more comparable treatments is recommended at a higher level (e.g., for an entire health plan or a large geographic region) level

- Negotiate treatment prices for an organization

- Advise decision makers regarding appropriate use of a treatment based on clinical factors only (e.g., in the form of clinical treatment guidelines for a health plan or geographic area)

-Advise decision makers regarding whether a treatment is a good value for money (e.g., conduct or publish health technology assessments)

- Other (please specify, especially as it pertains to making prescribing or formulary decisions or advising those decision makers)

2. Does PRO data impact decision making when considering one therapy over another?

Yes/no; if yes, rank the following 1-6:

-CNS: Neurology and Neurodegenerative

- CNS: Psychiatry

- Oncology

- Diabetes/Metabolic Disease

- Autoimmune Disorders

- "Lifestyle" (e.g., obesity, smoking)

Select the best response:

- Same for both types of trials

- Higher regards/more useful information from registration trials

- Higher regards/more useful information from postmarketing trials

- Other (please specify)

4. How frequently do you use PRO study data for risk-sharing agreements/risk-sharing schemes?

5. What is the level of importance of PRO data for market access for new oncology treatments in your health care system?

6. To what extent do PRO label claims increase your likelihood of paying for a treatment?

7. Which has more impact on decision making for a new treatment, PRO data in the label or PRO data in a peer-reviewed publication?

8. How important is full validation of the PRO measures in your use of PRO information in decision making?

9. To what extent do data collected through PROs that have been newly developed and may not have full validation influence your decision making?

10. What characteristics should a PRO measure for a treatment in oncology have in order to support market access and HTA?

\begin{tabular}{l|c}
\hline Please respond using a scale of 1 to 7 where 1 means "never" and 7 means "always" & 24 \\
\hline $\begin{array}{l}\text { Please respond using a scale of } 1 \text { to } 7 \text { where } 1 \text { means "not important" and } 7 \text { means } \\
\text { "extremely important" }\end{array}$ & 23 \\
\hline Please respond using a scale of 1 to 7 where 1 means "never" and 7 means "always" & 23 \\
\hline $\begin{array}{l}\text { Indicate label or publication } \\
\text { "extremely important" }\end{array}$ & 23 \\
\hline $\begin{array}{l}\text { Please respond using a scale of } 1 \text { to } 7 \text { where } 1 \text { means "not important" and } 7 \text { means } \\
\text { "extremely important" }\end{array}$ & 23 \\
\hline $\begin{array}{l}\text { Select all that apply and rate by level of importance: } \\
\text { - Developed per FDA PRO guidance document } \\
\text { - Frequently used in oncology or for a specific type of cancer } \\
\text {-Validated in target disease population } \\
\text { - Validated in country-specific populations } \\
\text { - Adopted by key opinion leaders } \\
\text { - Data from measure published in peer-reviewed journals } \\
\text { - Results supported by minimally clinically important difference estimates } \\
\text { - Results in alignment with objective measure results (e.g., laboratory value, clinician } \\
\text { rating) } \\
\text { • Other (please specify) }\end{array}$ & 23 \\
\hline
\end{tabular}

Number ${ }^{a}$

26 


\section{APPENDIX A Survey Questions (continued)}

Question

11 a. Are you aware of oncology products that received favorable reimbursement decisions because of PRO data?

b. Conversely, are you aware of examples of oncology products that did not receive a favorable reimbursement decision because they did not include a PRO?

12. a. How useful are PROs collected postprogression for an oncology therapeutic?

b. How long should postprogression data be collected?

13. In what types of cancers would it be most useful to collect PRO data while the cancer is progressing?

Yes/no; if yes, please indicate

Response Option

Number ${ }^{\mathrm{a}}$

-

Please rate on a scale of 1 to 7 where 1 means "not at all useful" and 7 means "extremely useful"

\begin{tabular}{l|l} 
Please rate the cancer indications on a scale of 1 to 7 where 1 means "not at all useful" & 20 \\
and 7 means "extremely useful":
\end{tabular}

- Lung (non-small cell lung cancer)

- Breast

- Bladder

- Hematological

- Other (please specify)

14. What type of data (PRO or other types of data) would be convincing for supporting reimbursement of oncology treatments that have stopped preventing the cancer from progressing (postprogression)?

Please respond using a sca

"extremely important":

- Stability of disease

- Improvement of HRQOL

- Improvement in symptom severity or frequency (e.g., cough, fatigue)

- Improvement in functional status (e.g., physical, social, emotional)

- Slower rate of functional deterioration compared with control/comparator

- Other (please specify)

15. How influential are the following sources of PRO data for market access decision making?

\begin{tabular}{l|l} 
Please respond using a scale of 1 to 7 where 1 means "no impact at all" and 7 means a & 20 \\
"very high impact" to market access decision making &
\end{tabular}

- Peer-reviewed publication

- PRO data source from registration trials

-PRO data source from postmarketing trials

- Professional conference presentation (e.g., ASCO)

- Treatment guideline from clinician organization

- Additional documentation provided by the manufacturer

- Manufacturer's website for the product

- Patient advocacy organization

- Social media

- Other

16. If you impart one piece of advice for pharmaceutical manufacturers with respect to communicating PRO evidence to payer decision makers, what would it be?

a Number of respondents who answered the question.

bTwelve participants responded "yes" and provided a ranking.

ASCO=American Society of Clinical Oncology; CNS =central nervous system; FDA=U.S. Food and Drug Administration; HRQOL=health-related quality of life;

$H T A=$ health technology assessment; $P R O=$ patient-reported outcome . 
APPENDIX B Influence of PRO Label Claim on Payer Decision Making

United States $(n=15)$

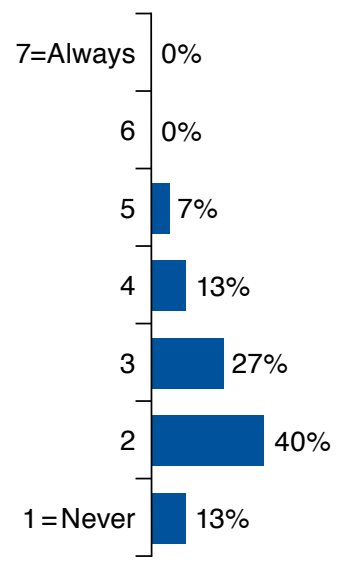

Ex-United States $(n=8)$

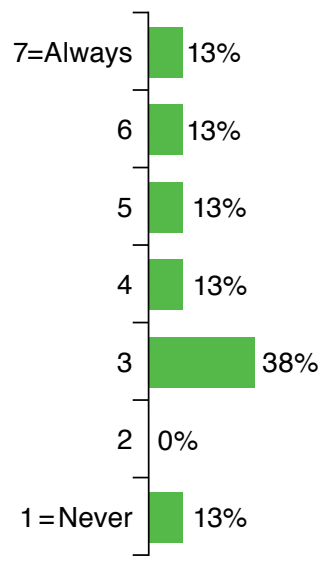

Global $(n=23)$

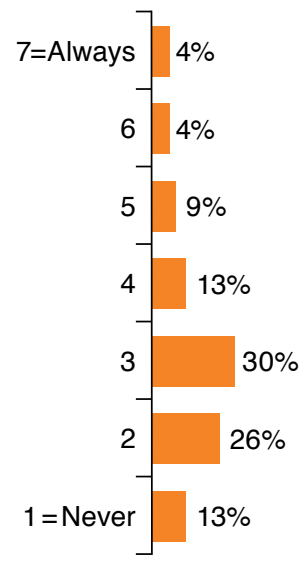

